\title{
Combined Endovascular/Neurosurgical Therapy of Blister-Like Distal Internal Carotid Aneurysms
}

\author{
D.M. Pelz, G.G. Ferguson, S.P. Lownie, E. Kachur
}

\begin{abstract}
Objective: To describe a novel therapeutic strategy for the treatment of "blister-like" aneurysms of the distal internal carotid artery (ICA). Direct surgical treatments of these fragile lesions have been associated with generally poor outcomes. Methods: Two consecutive patients presenting with acute subarachnoid hemorrhage from "blister-like" aneurysms were treated with preliminary balloon occlusion of the ICA, followed by surgical trapping of the ICA beyond the aneurysm. Results: The treatment resulted in complete thrombosis of both aneurysms with no clinical complications. Conclusion: This combined endovascular-neurosurgical approach offers a controlled, safer alternative to primary surgical therapy of "blister-like" aneurysms.
\end{abstract}

RÉSUMÉ: Thérapie combinée endovasculaire et neurochirurgicale des anévrismes sacciformes de la carotide interne distale. Objectif: Décrire une stratégie thérapeutique nouvelle pour le traitement des anévrismes sacciformes de la carotide interne distale (CID). Le traitement chirurgical direct de ces lésions fragiles a été associé à des résultats généralement défavorables. Méthodes: Deux patients consécutifs présentant une hémorragie sous-arachnoïdienne aiguë provenant d'anévrismes sacciformes ont été traités par occlusion préliminaire par ballon de la CID, suivie d'un piégeage de la CID au-delà de l'anévrisme. Résultats: Le traitement a provoqué la thrombose complète des deux anévrismes sans complication clinique. Conclusion: Cette approche endovasculaire et neurochirurgicale combinée offre une alternative contrôlée, plus sûre que le traitement chirurgical primaire, des anévrismes sacciformes.

Can. J. Neurol. Sci. 2003; 30: 49-53

The term "blister-like" aneurysm has been used to describe a small, sessile, hemispherical expansion of the supraclinoid segment of the distal internal carotid artery (ICA). ${ }^{1-4}$ These lesions may be the source of subarachnoid hemorrhage (SAH) and are characterized by very fragile walls, poorly defined necks and histology distinct from saccular aneurysms, making surgical exploration and clipping hazardous. ${ }^{1-6}$ Therapeutic strategies have been variable, including attempted clipping, wrapping, clipping and wrapping, ICA occlusion, trapping, and endovascular coiling with no consensus being reached on the optimal approach. ${ }^{1,4-7}$ We describe two cases of acute $\mathrm{SAH}$ caused by these lesions, treated successfully and uneventfully by proximal ICA balloon occlusion followed by surgical clipping of the ICA beyond the aneurysm.

\section{Patient 1}

A 34-year-old woman suddenly collapsed with a rapidly decreasing level of consciousness requiring emergency intubation. A computed tomographic (CT) head scan showed acute SAH with slightly more blood seen in the right side of the suprasellar cistern (Figure 1). Cerebral angiography performed on the same day showed a sessile, broad-based aneurysm of the supraclinoid segment of the right ICA and an abnormality of the cervical segment, suspicious for fibromuscular dysplasia (Figure 2). No other lesions were identified and the ICA aneurysm was considered to be the source of SAH. Due to the patient's poor clinical status and the known risks of surgical treatment of these aneurysms, immediate clipping was not attempted.

As the patient's level of consciousness improved and she was extubated, it was decided to perform a trial occlusion and, if tolerated, a permanent balloon occlusion of the right ICA. It was hoped that this might result in aneurysm thrombosis and, if not, subsequent surgery would be facilitated by the decreased pressure in the aneurysm and distal ICA. Her collateral supply to the ipsilateral hemisphere would also have already been determined in a controlled manner.

The patient tolerated trial occlusion of the cervical ICA for 30 minutes without incident, three days after admission. Permanent balloon occlusion was then performed. However, the subsequent angiogram

From the Department of Diagnostic Radiology and Nuclear Medicine (DMP, SPL); Department of Clinical Neurological Sciences (GGF, SPL, EK); London Health Sciences Centre, University Campus, University of Western Ontario, London, Ontario, Canada.

ReCeived March 26, 2002. AcCePted in Final FORM July 11, 2002. Reprint requests to: David M. Pelz, Department of Diagnostic Radiology, 339 Windermere Road, P.O. Box 5339, London, Ontario, Canada N6A 5A5 
showed continued filling of the aneurysm via right ophthalmic artery collaterals (Figure 3). The right posterior communicating artery (pcom) was the main collateral supply to the right middle cerebral artery (MCA). Her clinical condition fluctuated over the next week and a subsequent angiogram seven days later showed the development of moderate vasospasm but no change in aneurysm filling. The vasospasm was successfully managed medically. A follow-up study one week later showed resolution of the vasospasm with continued visualization of the aneurysm. It was then decided to proceed with surgical trapping of the aneurysm rather than direct clipping. A right pterional craniotomy was performed and exposure of the distal right ICA showed the aneurysm as a sessile expansion of the ICA, directed anteriorly and medially as the vessel exited beneath the anterior clinoid process. There was discoloration of the vessel wall near the hemorrhage site, with evidence for old clotted blood around the distal ICA. The pcom was located quite proximally and the ICA beyond it was normal in appearance. The petroclinoid ligament was partially divided to obtain proximal exposure of the ICA, and a bayonetted Sugita clip was used to clip the ICA just proximal to the pcom. Wrapping with muslin gauze was then performed. A postoperative angiogram showed no filling of the aneurysm from either the ophthalmic artery or pcom (Figure 4A,B). The patient made an excellent recovery with only some residual visual loss, from a previous subhyaloid hemorrhage, on discharge.

\section{Patient 2}

A 24-year-old male student experienced a sudden severe headache with nausea and vomiting followed by momentary collapse. On initial examination, apart from residual headache, he was completely intact neurologically. A CT head scan showed acute SAH with a right-sided predominance (Figure 5). Angiography performed the same day showed a small sessile aneurysm of the anterior wall of the supraclinoid right ICA, opposite the origin of a large pcom (Figure 6). An unusual fenestration with aneurysm was also identified in the petrous segment of the ICA.

There was no history of trauma. No other vascular lesions were seen. Due to the recent experience with Patient 1, it was again decided to proceed with trial and then permanent balloon occlusion of the ICA, followed by surgical trapping if necessary. Permanent balloon occlusion was successfully performed four days after admission, however the aneurysm continued to fill afterwards via the pcom (Figure 7A,B). Although the patient remained well, a transcranial Doppler and CT angiography indicated the development of moderate to severe vasospasm two days later which persisted for two weeks, when transcranial Dopplers returned to normal. Again, medical management of the vasospasm was successful. Surgery was then performed three weeks after admission. On exposure of the distal right ICA, a red, rounded aneurysm was identified, located diagonally across from the medially directed pcom. An angled clip was placed on the ICA just distal to the aneurysm and proximal to the pcom with the clip blades pointing away from the latter vessel. Absence of flow in the aneurysm and patency of the pcom and distal ICA were confirmed with a microDoppler probe. The patient remained well postoperatively and a subsequent angiogram showed no filling of the aneurysm (Figure 8).

\section{Discussion}

Unusual sessile aneurysms of the distal ICA were first described by Sundt and Murphy ${ }^{8}$ in 1969 and designated as "blister-like" in the Japanese literature by Takahashi et $\mathrm{al}^{9}$ in
1988 and in the English literature by Shigeta et $\mathrm{al}^{6}$ in 1992 . These lesions are not common, accounting for $0.4-1.4 \%$ of cerebral aneurysms ${ }^{1,3}$ Many terms have been used to describe them, including "dorsal wall", "anterior wall", "superior wall", and "internal carotid trunk". ${ }^{1,4}$ They are characterized by several features which distinguish them from classical saccular aneurysms. They are not located at arterial bifurcations and they tend to arise on the anteromedial surface of the proximal supraclinoid ICA. The walls of these lesions are thin, fragile and easily lacerated at surgery. ${ }^{10}$ Pathologically, they are different from true aneurysms. They not only lack an internal elastic lamina and media but the gap in the internal elastic lamina, normally covered by thickened collagenous adventitia in classical berry aneurysms ${ }^{11}$ is not observed. There is usually no evidence for inflammation or dissection. ${ }^{2}$ Although speculative at present, they are thought to represent lacerations of the carotid wall due to degeneration of the internal elastic lamina. Rapid angiographic progression to a more classical saccular appearance has been described, likely the result of clot organization over the intimal defect. ${ }^{1,5,7}$

The pathogenesis of these aneurysms is uncertain. Aneurysms at nonbranching sites have been thought to result from atherosclerotic weakening of the arterial wall ${ }^{11}$ and premature atherosclerotic changes in the distal ICA have been reported. ${ }^{5}$ Some authors have noted an association with proximal ICA dissection, hypertension, a younger age group, and female predominance. ${ }^{4}$ Hemodynamic stresses at a tight arterial curve may also play a role. ${ }^{1,3}$

Treatment strategies and therapeutic results have been variable. In the largest series of 40 such aneurysms, Ogawa et al ${ }^{4}$ directly clipped 21 , circumferentially clipped ten after wrapping, wrapped four, and surgically trapped seven, two of which required preliminary superficial temporal-MCA bypass. Significant intra- and postoperative bleeding occurred in 15 patients due to vessel laceration and aneurysm growth, resulting in eight deaths. Three patients died from massive infarction following ICA ligation. Despite the dangers encountered during surgery, often resulting from preliminary dissection and clot removal around the aneurysm, the authors still believed that clipping of a circumferentially wrapped aneurysm was the best therapeutic strategy. The need for cervical ICA control was emphasized and preliminary endovascular ICA occlusion was mentioned but not discussed. Abe et al ${ }^{1}$ treated six patients, three of whom had direct clipping with two arteries rupturing intraoperatively leading to fatal outcomes. Two aneurysms were wrapped but both grew on subsequent angiograms, leading to clipping in one and endovascular ICA occlusion in another. One patient was initially treated conservatively, followed by balloon occlusion of the cervical ICA following superficial temporalMCA bypass, resulting in thrombosis of the aneurysm. There were three excellent results, one after clipping and two after ICA occlusion. Again, the authors favoured initial surgical clipping, with or without wrapping. They believed that surgery was better in the chronic than in the acute stage and that endovascular ICA occlusion may be useful to reduce pressure in the aneurysm and promote thrombosis.

Nakagawa et $\mathrm{al}^{3}$ reported on eight surgically treated patients, three of whom bled intra-operatively and one fatally postoperatively. Surgery was still the preferred treatment and the 
Figure 1: Patient one: CT head scan showing acute subarachnoid hemorrhage, with a slight right-sided predominance in the suprasellar cistern.
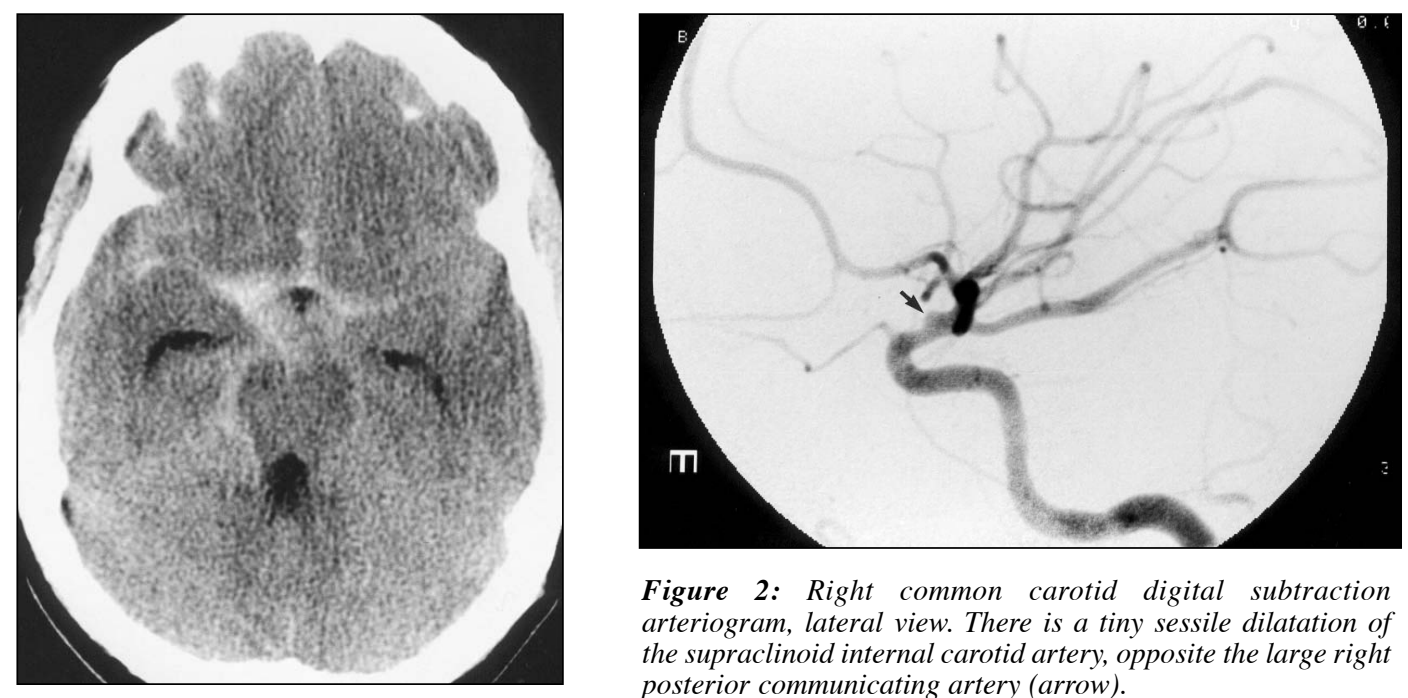

Figure 2: Right common carotid digital subtraction arteriogram, lateral view. There is a tiny sessile dilatation of the supraclinoid internal carotid artery, opposite the large right posterior communicating artery (arrow).

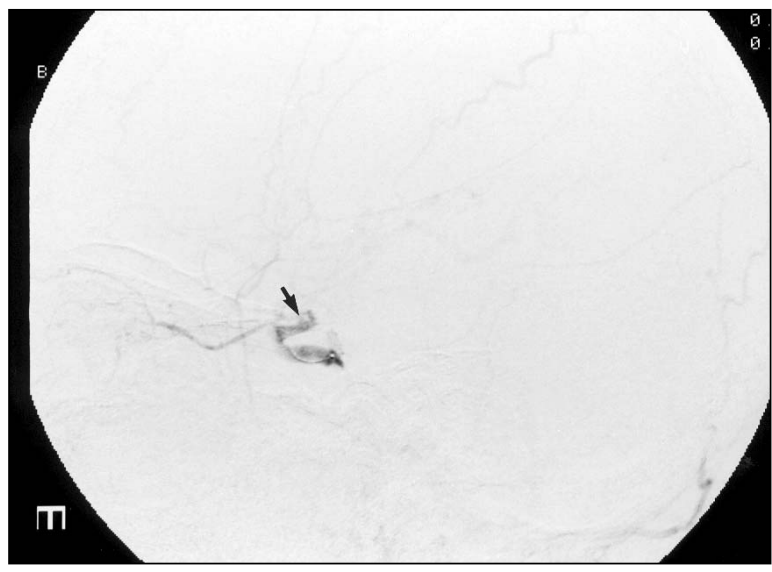

Figure 3: Right common carotid digital subtraction arteriogram lateral view following balloon occlusion of the internal carotid artery. The aneurysm continues to fill (arrow) via ophthalmic artery collaterals.

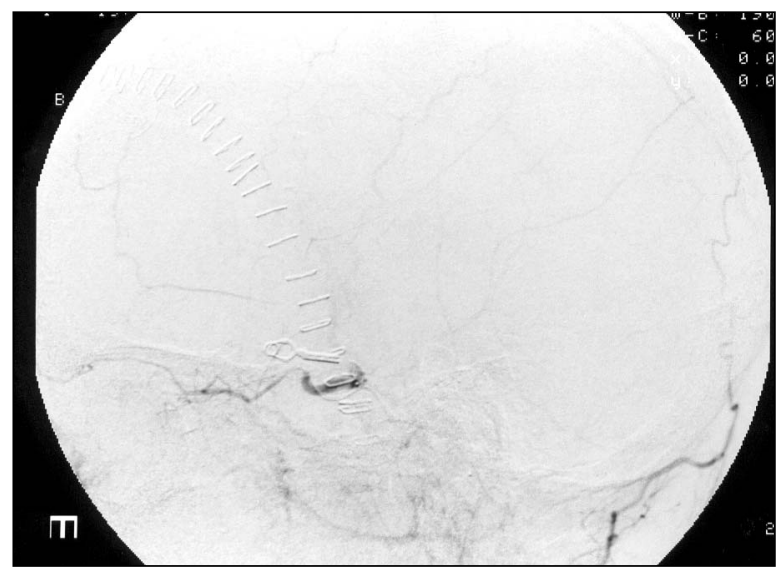

Figure 4A: Right common carotid digital subtraction arteriogram, lateral view following surgical clipping of distal internal carotid artery. There is filling of the proximal cavernous internal carotid artery via ophthalmic artery collaterals but there is no filling of the aneurysm.

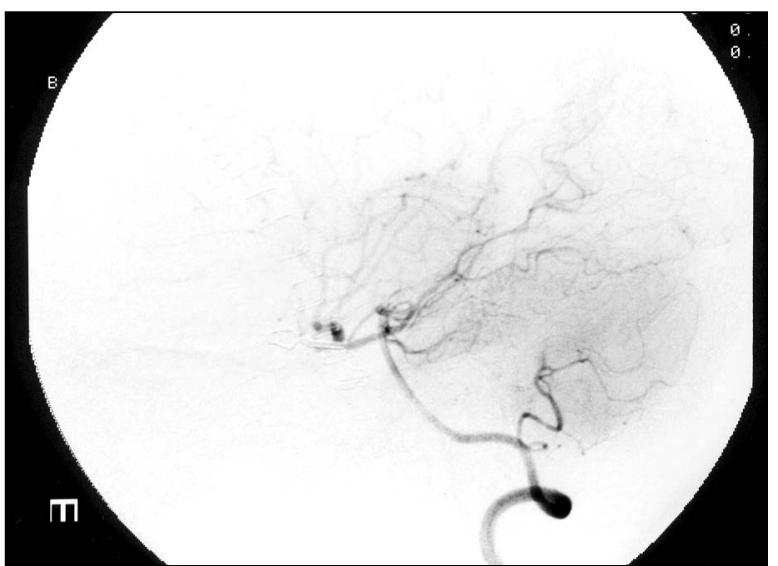

Figure 4B: Left vertebral digital subtraction arteriogram, lateral view postoperatively shows good filling of the right pcom and right middle cerebral artery but no filling of the aneurysm. 

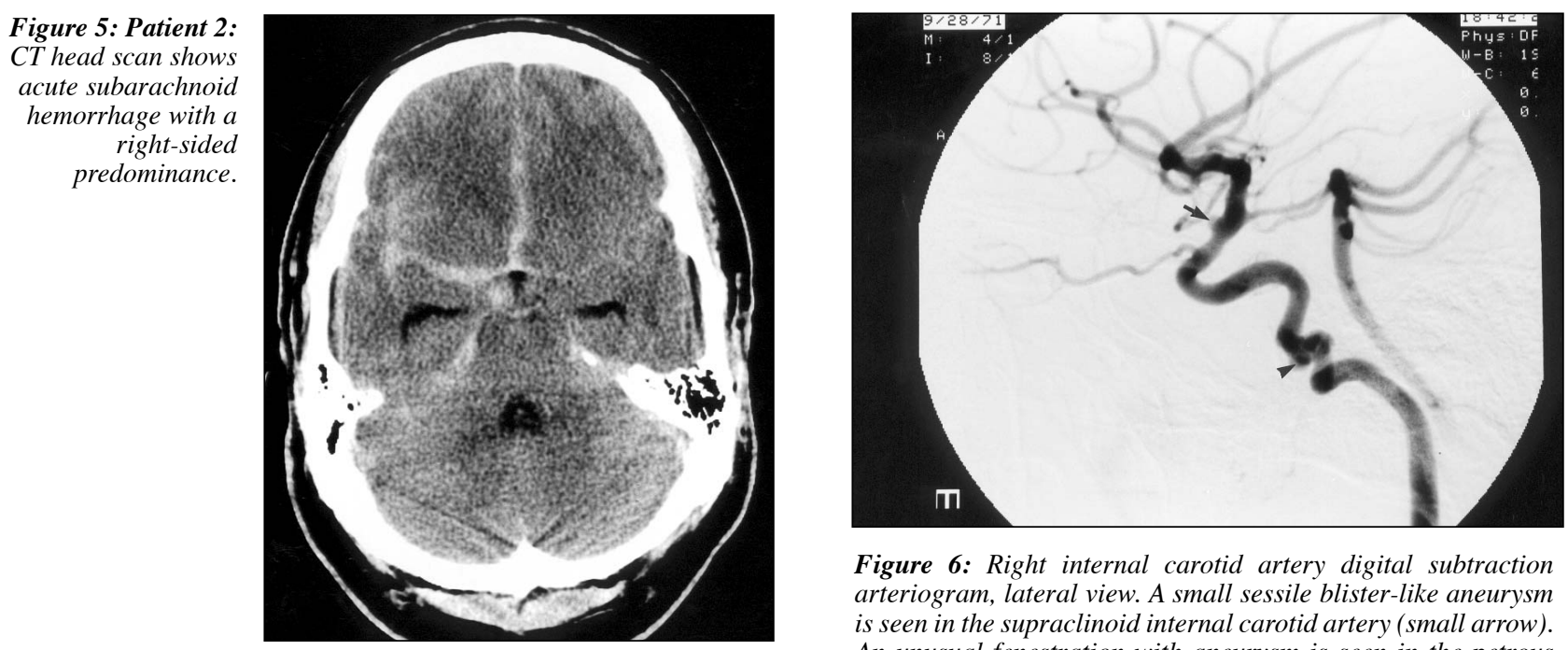

Figure 6: Right internal carotid artery digital subtraction arteriogram, lateral view. A small sessile blister-like aneurysm is seen in the supraclinoid internal carotid artery (small arrow). An unusual fenestration with aneurysm is seen in the petrous internal carotid artery (arrowhead).

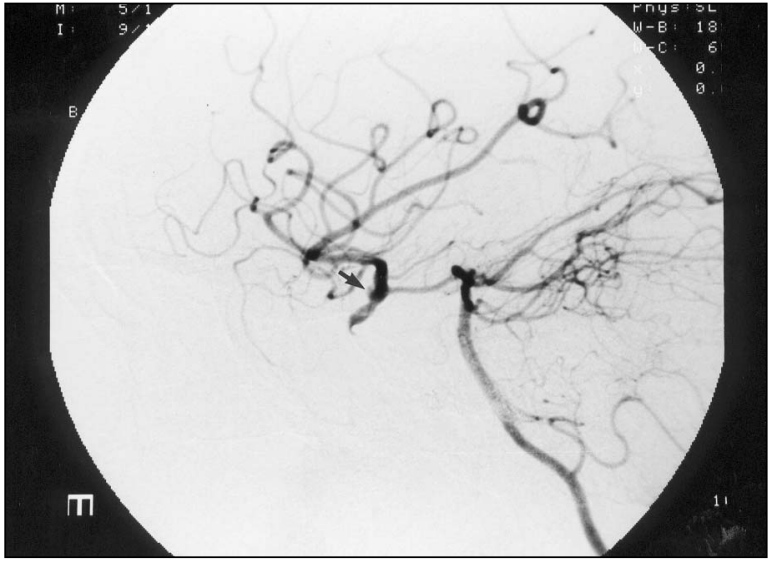

Figure 7A: Left vertebral digital subtraction arteriogram, lateral view post balloon occlusion of the right internal carotid artery. The aneurysm (arrow) continues to fill via the right pcom.

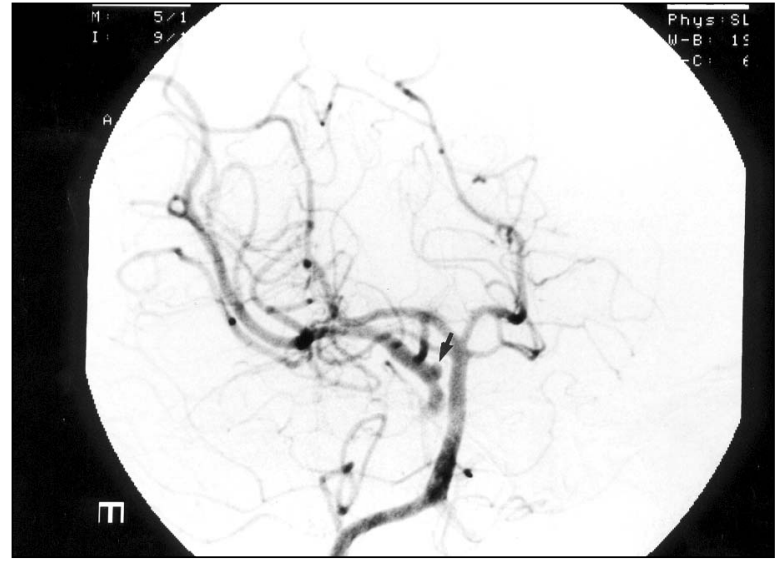

Figure 7B: An AP oblique projection from the same study again shows filling of the aneurysm (arrow).

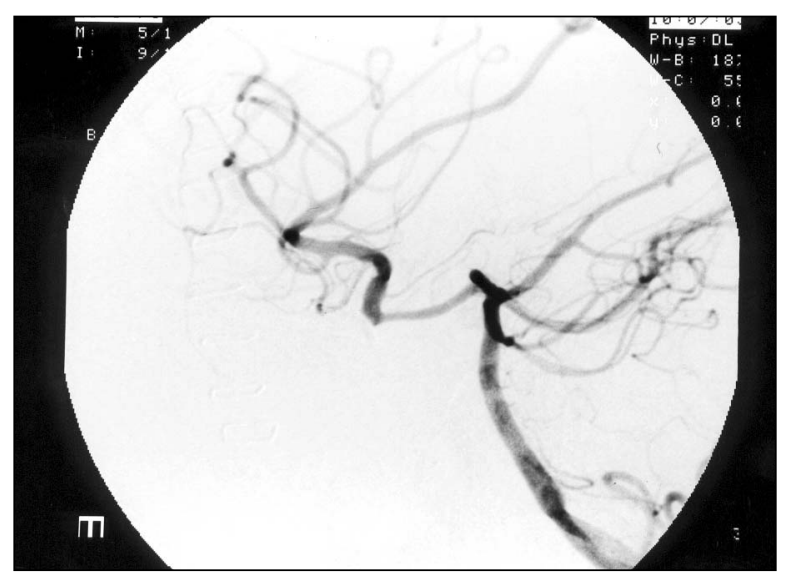

Figure 8: Left vertebral digital subtraction arteriogram, lateral view, post surgical clipping of the distal internal carotid artery shows patency of the pcom, no filling of the aneurysm and good filling of the right middle cerebral artery. 
authors stressed the need to position the clip blades parallel to the parent vessel to avoid laceration. They speculated on potential benefits of induced hypotension, temporary ICA occlusion and reinforcment of the arterial wall with plastic adhesives, muscle, cellulose fabric, and fibrin glue. Redekop et $\mathrm{al}^{5}$ described five patients, all of whom had surgery. There were two intraoperative ruptures and one severe hemorrhage three days postoperatively. One patient suffered a large MCA stroke after surgical trapping of the ICA. Again, the need for careful aneurysm dissection and control of the cervical ICA were emphasized. Brief mention was made of potential endovascular therapy with stents and coils. McNeely et $\mathrm{al}^{7}$ reported on one patient successfully treated with Guglielmi detachable coils following unsuccessful surgery and acute aneurysm enlargement.

In our two patients, we were reluctant to proceed with immediate surgery due to the known fragility of these lesions and the hazards documented in the literature. The necessity for proximal ICA control led us to consider initial endovascular balloon occlusion. This would not only assess the collateral potential to the ipsilateral hemisphere in a controlled fashion, but would also provide some protective effect by acutely decreasing pressure in the aneurysm and in the most optimistic scenario, promote thrombosis in the aneurysm itself, as has been previously reported. ${ }^{1}$ We did not expect that the balloon occlusion alone would necessarily thrombose the aneurysm. It was decided not to attempt balloon or coil placement in the cavernous or supraclinoid segments of the ICA because of the known fragility of the vessel in this condition. There is also a danger of occluding branches of the ICA such as the pcom, the anterior choroidal artery and smaller perforators, as both balloons and coils have length and precise, focal placement of these devices may be technically difficult.

We believe that the two procedures, preliminary balloon occlusion and craniotomy with surgical clipping were justified to ensure precise, less hazardous trapping of the aneurysm. We knew that preliminary sacrifice of the ICA may not result in aneurysm thrombosis, however we believed that it was justified to eliminate the need for surgical manipulation of a fragile, diseased arterial segment. The hazards of a direct surgical approach to this region have been well-documented. ${ }^{10}$ Failing complete aneurysm thrombosis, we thought that subsequent surgery trapping of the ICA beyond the aneurysm would thus be facilitated by reducing the risk of uncontrolled intraoperative hemorrhage.

Vasospasm was successfully managed medically in both patients despite early carotid occlusions. We were fortunate that endovascular balloon angioplasty for vasospasm was not necessary which would have been precluded by the preliminary balloon occlusion. Intra-arterial papaverine therapy may have been possible through the posterior communicating artery.

At surgery, it was decided to occlude the ICA just beyond the aneurysm but proximal to large pcoms rather than risk direct clipping, as the vessel walls were clearly abnormal in both cases.
Excellent angiographic and clinical results were observed with this staged, conservative approach. We were also fortunate that both patients tolerated balloon occlusion of the internal carotid artery and did not re-bleed from their aneurysms. For patients who do not tolerate trial occlusion, alternate treatment strategies must be considered, including preliminary superficial temporal artery to MCA bypass prior to surgery. This treatment may also be inappropriate for patients with poor neurologic grade in whom tolerance of balloon occlusion cannot be adequately assessed. The long-term risk of stroke in young patients with carotid balloon occlusion is also not known.

In conclusion, "blister-like" aneurysms of the supraclinoid ICA are unusual, fragile lesions which are distinct from saccular aneurysms. Direct surgical clipping is hazardous and although reported treatment strategies are variable, most series have emphasized the need for proximal ICA control. We describe two patients in which preliminary endovascular balloon occlusion of the ICA was performed, followed by surgical clipping of the ICA distal to the diseased segment, resulting in excellent angiographic and clinical outcomes. This treatment strategy is proposed as one of many possible therapies for these challenging lesions.

\section{REFERENCES}

1. Abe M, Tabuchi K, Yokoyama H, Uchino A. Blood blister-like aneurysms of the internal carotid artery. J Neurosurg 1998;89:419-424.

2. Ishikawa T, Nakamura N, Houkin K, Nomura M. Pathological consideration of a "blister-like" aneurysm at the superior wall of the internal carotid artery: case report. Neurosurgery 1997;40(2):403-405.

3. Nakagawa F, Kobayashi S, Takamae T, Sukita K. Aneurysms protruding from the dorsal wall of the internal carotid artery. J Neurosurg 1986;65:303-308.

4. Ogawa A, Suzuki M, Ogasawara K. Aneurysms at non-branching sites in the supraclinoid portion of the internal carotid artery: internal carotid trunk aneurysms. Neurosurgery 2000;47(3):578583.

5. Redekop GJ, Woodhurst B. Unusual aneurysms of the distal internal carotid artery. Can J Neurol Sci 1998;25:202-208.

6. Shigeta H, Kyoshima K, Nagakawa F, Kobayashi S. Dorsal internal carotid aneurysms with special reference to angiographic presentation and surgical management. Acta Neurochir (Wien) 1992;119:42-48

7. McNeely PD, Clarke DB, Baxter B, Vandorpe RA, Mendez I. Endovascular treatment of a "blister-like" aneurysm of the internal carotid artery. Can J Neurol Sci 2000;27:247-250.

8. Sundt TM, Murphy F. Clip grafts for aneurysm and small vessel surgery. J Neurosurg 1969;31:59-71.

9. Takahashi A, Suzuki J, Fujiwara S, Mizoi K, Yoshimoto T. Surgical treatment of chimame (blood blister-like) aneurysm at the $\mathrm{C} 2$ portion of internal carotid artery (in Japanese). Surg Cereb Stroke 1988; 16:72-77.

10. Tanda Y, Hongo K, Tada $\mathrm{T}$, et al. Radiometric analysis of paraclinoid carotid artery aneurysms. J Neurosurg 2002;96:649653.

11. Stehbens WE. Etiology of intracranial berry aneurysms. J Neurosurg 1989;70:823-831. 